\title{
Influence of the Characteristics of a Workpiece on the Slicing Characteristics Including Tool Wear
}

\author{
Satoshi Sakamoto ${ }^{1}$, Masaya Gemma ${ }^{1}$, Yasuo Kondo², Kenji Yamaguchi ${ }^{3}$, Mitsugu Yamaguchi ${ }^{4}$, and Takao Yakou ${ }^{1}$ \\ ${ }^{1}$ Yokohama National University, 79-2 Tokiwadai, Hodogaya-ku, Yokohama, Japan \\ ${ }^{2}$ Yamagata University, 4-3-16 Jonan, Yonezawa, Japan \\ ${ }^{3}$ Yonago National College of Technology, 4448 Hikona-cho, Yonago, Japan \\ ${ }^{4}$ Salesian Polytechnic, 4-6-8 Oyamagaoka, Machida, Japan
}

\begin{abstract}
Multi-wire saws with a diamond electrodeposited wire tool are widely used to slice hard and brittle materials. The properties of the materials significantly affect the saw's performance in terms of slicing quality, efficiency, and accuracy. In this study, the effects of the material properties of workpieces on wire tool performance and tool wear are described. The main conclusions are as follows: the higher the hardness and toughness of the workpiece material, the longer the slicing time and greater the damage to the wire tool. The brittleness of the workpiece adversely affects the thickness variation more than its hardness. In addition, the frequency of chipping mainly depends on the hardness of the workpiece, whereas the chipping size mainly depends on the toughness of the workpiece.
\end{abstract}

\section{Introduction}

Multi-wire sawing is an excellent method for slicing hard and brittle materials such as semiconductors and optical materials [1]. Recently, multi-wire sawing using a diamond electrodeposited wire tool has been adopted as the preferred slicing method for high-hardness materials [1]. In addition, new types of wire tools and slicing devices are being developed [2-4]. However, the occurrence of microcracks on the workpiece due to slicing adversely affects slicing efficiency and accuracy. Therefore, it is important to clarify the relationship between the workpiece and slicing characteristics.

We have previously clarified the influence of the mechanical properties of workpieces on the polishing and grooving characteristics $[5,6]$. In this study, we describe the relationship between the mechanical properties of workpieces and slicing characteristics, including tool wear.

\section{Experimental procedure}

Figure 1 shows a schematic illustration of the experimental apparatus. The multi-wire saw used in this study is a wire reciprocating type. Therefore, one drum simultaneously controls the feeding and rewinding of a wire tool.

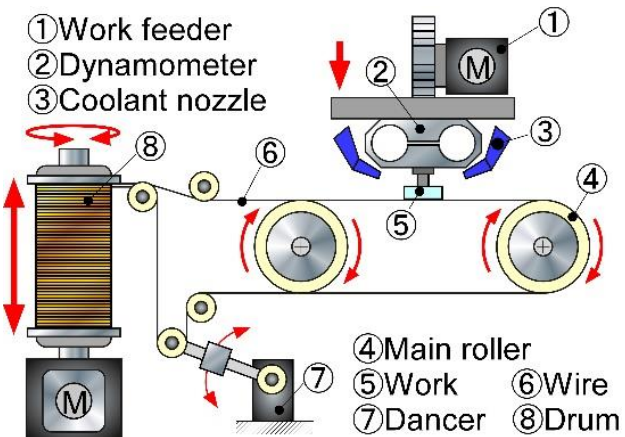

Figure 1. Schematic of the multi-wire saw device used in this study.

Table 1 shows the materials used and main experimental conditions. The multi-wire saw included a diamond electrodeposited wire tool with a core diameter of $100 \mu \mathrm{m}$, an outer diameter of $143 \mu \mathrm{m}$, and tension set at $14.6 \mathrm{~N}$. Borosilicate glass (TEMPAX), silicon (single crystal, polycrystalline), and sapphire were used as workpieces with dimensions of $20 \mathrm{~mm} \times 20 \mathrm{~mm} \times 5 \mathrm{~mm}$. A glycol-based water-soluble coolant supplied at 50 $\mathrm{mL} / \mathrm{min}$ was used as the working fluid. The running speed of the wire tool was $100 \mathrm{~m} / \mathrm{min}$, and the feed speed of the workpiece was $0.8 \mathrm{~mm} / \mathrm{min}$. Table 2 shows the hardness and toughness value of the TEMPAX, silicon, and sapphire 
workpieces [7, 8]. Silicon is a numerical value of a single crystal.

Table 1. Experimental conditions and material used.

\begin{tabular}{ll}
\hline Wire tool & \\
Core diameter & $100[\mu \mathrm{m}]$ \\
Abrasives & Diamond \\
Grain size & $20-30[\mu \mathrm{m}]$ \\
Outer diameter & $143[\mu \mathrm{m}]$ \\
Running speed & $100[\mathrm{~m} / \mathrm{min}]$ \\
Tension & $14.6[\mathrm{~N}]$ \\
Usage length & $250[\mathrm{~m}]$ \\
\hline Workpiece & \\
Material & Borosilicate glass (TEMPAX) \\
& Silicon $($ Mono-Si, Poly-Si) \\
& Sapphire \\
Dimension & $20 \times 20 \times 5\left[\mathrm{~mm}{ }^{3}\right]$ \\
Feed speed & $0.8\left[\mathrm{~mm} / \mathrm{min}^{2}\right]$ \\
\hline
\end{tabular}

Coolant

Glycol-based water-soluble working fluid

Supply amount $50[\mathrm{~mL} / \mathrm{min}]$

Table 2. Vickers hardness and fracture toughness of workpieces.

\begin{tabular}{lccc}
\hline Workpiece & TEMPAX & $\begin{array}{c}\text { Silicon } \\
\text { (Mono-Si) }\end{array}$ & Sapphire \\
\hline $\begin{array}{l}\text { Vickers } \\
\text { hardness }\end{array}$ & 6.2 & 10.0 & 23.0 \\
{$[\mathrm{GPa}]$} & & & \\
\hline $\begin{array}{l}\text { Fracture } \\
\text { toughness } \\
{[\mathrm{MPa}]}\end{array}$ & 0.7 & 1.14 & 2.1 \\
\hline
\end{tabular}

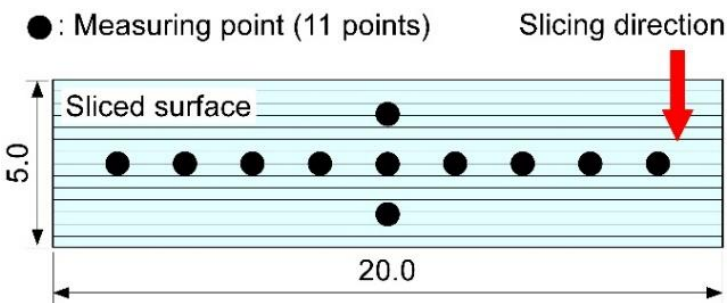

Figure 2. Thickness measuring points.

Figure 2 shows the measurement position of the wafer thickness. The thickness of the wafer was measured with a micrometer at 11 points, as shown in the figure.

\section{Experimental results and discussion}

\subsection{Slicing time of workpieces}

Figure 3 shows a comparison of the slicing time required until slicing is completed. Slicing time from the shortest to longest are ranked as follows: TEMPAX, silicon, and sapphire. This result is affected by the hardness and toughness of the materials, as shown in Table 2. Since sapphire had the highest hardness and toughness values among the three workpieces, slicing was more difficult and took much longer, whereas TEMPAX, which had the lowest hardness and toughness values, was easier to slice and took a much shorter time. No significant difference in slicing time was found between the single crystal silicon and polycrystalline silicon workpieces.

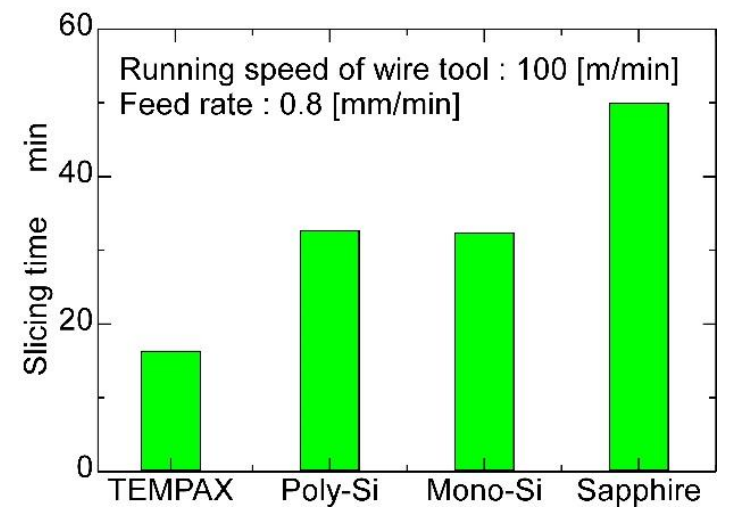

Figure 3. Slicing time for TEMPAX, silicon, and sapphire workpieces.

\subsection{Thickness variation of TEMPAX, silicon, and sapphire wafers}

Figure 4 shows a comparison of thickness variation of the TEMPAX, silicon, and sapphire workpiece. Sapphire has smaller thickness variation than the other workpieces. In general, the slicing of materials possessing a high value of hardness tends to cause the wire tool to stray. However, sapphire has both a high value of hardness and the highest value of toughness in the workpieces used in this study. Consequently, excessive microfracture of the workpiece and the wandering of the wire tool were suppressed. Thus, the slicing of sapphire requires higher slicing time, but the slicing accuracy is good. On comparing TEMPAX and sapphire, it is evident that the thickness variation of sapphire is smaller. In other words, the brittleness of the workpiece adversely affects the thickness variation more than its hardness.

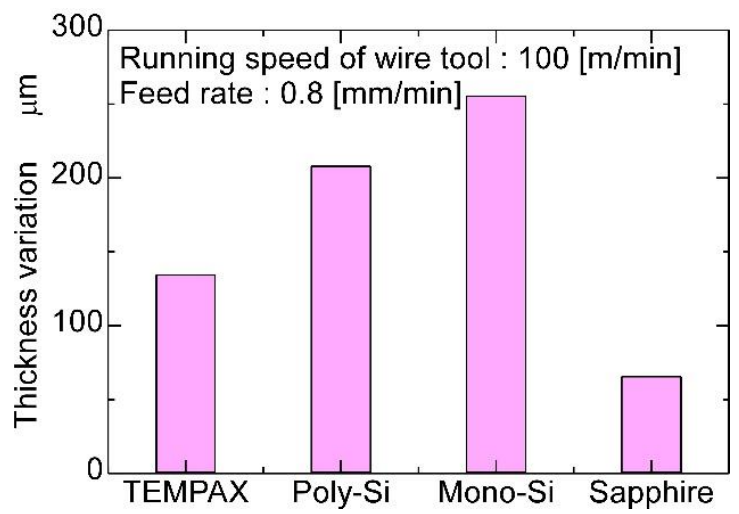

Figure 4. Comparison of thickness variation of the wafer. 


\subsection{Sliced surface of TEMPAX, silicon and sapphire wafers}

Figure 5 contains images of the sliced surfaces for TEMPAX, silicon, and sapphire. Saw marks from the wire tool are apparent on the sliced surface of all workpieces. However, the sliced surface of sapphire shows slightly better properties. The toughness of the workpiece affects the properties of the sliced surface, i.e., a workpiece with higher toughness suffers less damage to its sliced surface than a workpiece with low toughness.
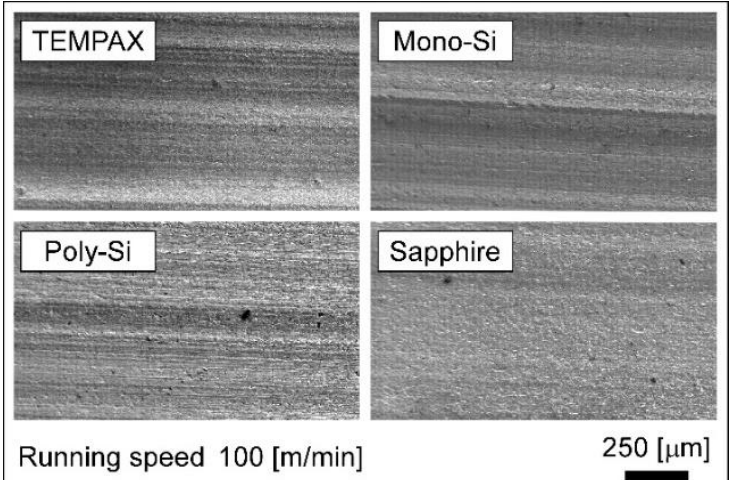

Running speed $100[\mathrm{~m} / \mathrm{min}]$

$50[\mu \mathrm{m}]$

Figure 5. Examples of sliced surfaces.

Figure 6 shows the scanning electron microscopy (SEM) images of the TEMPAX, silicon, and sapphire workpieces along the edge of the wafer. The edge of the sapphire workpiece is relatively better than the edge of the other workpieces.

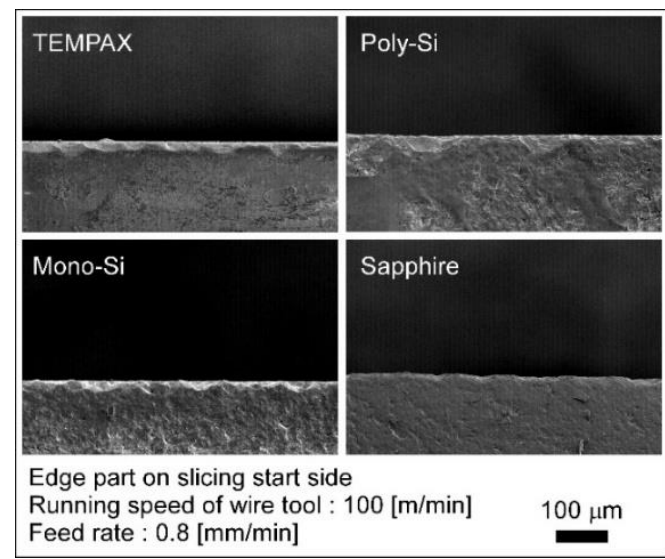

Figure 6. SEM images of the edge part of the wafer.
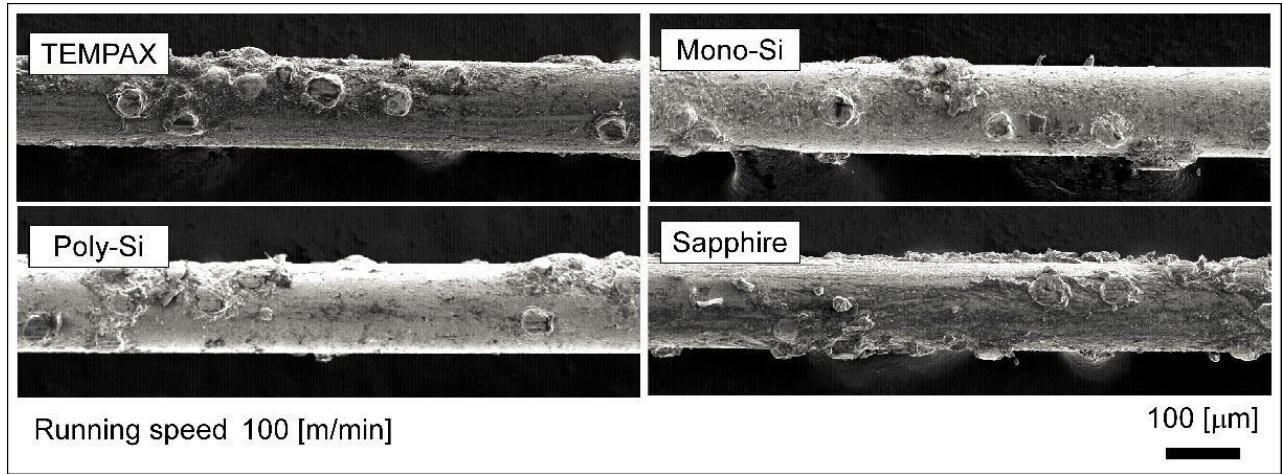

Running speed $100[\mathrm{~m} / \mathrm{min}]$
Figure 7 shows the chipping size and number of chipping that occurred at the edge of the wafer. The measurement distance of chipping is $600 \mu \mathrm{m}$. The indents that exceed the threshold are analyzed using the vector image analysis software and are measured as the number of chipping in this study. The threshold was set at $1.50 \mu \mathrm{m}$. The number of chipping on the edge of the sapphire and polycrystalline silicon workpieces is slightly larger. However, the size of chipping on the sapphire is very small. Conversely, the edge of TEMPAX has a smaller number of chipping, but its chipping size is large. The frequency of chipping mainly depends on the hardness of the workpiece, whereas the chipping size mainly depends on the toughness of the workpiece. In other words, the hard material tends to slightly increase the number of chips and the high toughness material tends to slightly reduce the chip size.

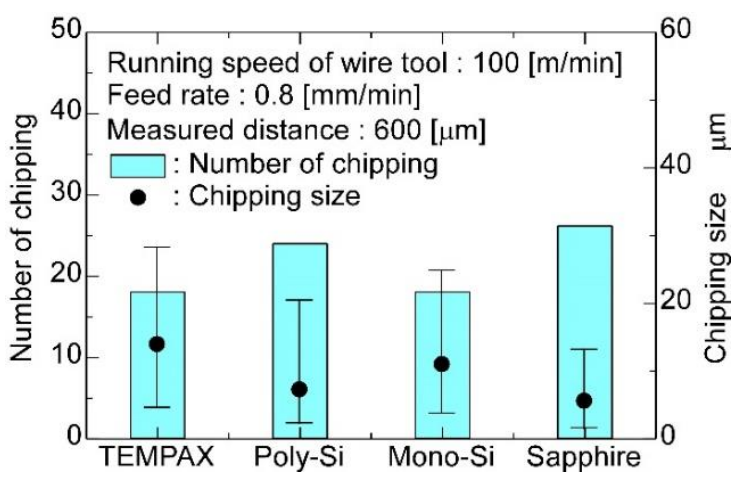

Figure 7. Comparison of number and size of chipping.

\subsection{Influence on the damage of the wire tool}

Figure 8 shows the wire tools after slicing the workpieces. Damage such as falling-off of abrasives occurred in all the wire tools for all workpieces. However, the wire tool used for slicing the TEMPAX appeared to be less damaged because the slicing time was shorter. On the contrary, the wire tool used to slice sapphire showed significant damage on both the surface and the core wire.

Figure 8. Wire tool damage after slicing. 


\section{Conclusions}

We experimentally investigated the influence of the properties of workpieces on the slicing characteristics, including tool wear, of workpieces. The main conclusions obtained from this study are as follows. The hardness and brittle behavior of the work materials affect the slicing characteristics. Higher the hardness and toughness of the workpiece, longer is the slicing time required and greater is the damage to the tool. Especially, the wire tool used to slice sapphire with high hardness and toughness suffers significant damage. The brittleness of the workpiece adversely affects the wafer thickness variation more than its hardness. Therefore, in sapphire having high toughness, the wafer thickness variation is small. The workpiece with higher toughness suffers less damage to its sliced surface than the workpiece with low toughness. In addition, the hard material tends to slightly increase the number of chips and the high toughness material tends to slightly reduce the chip size.

\section{Acknowledgments}

We thank Instrumental Analysis Center of Yokohama National University for their support and cooperation. This work was supported by JSPS KAKENHI Grant Number $16 \mathrm{~K} 06005$.

\section{References}

[1] H Wu 2016 Precis. Eng. 431

[2] Kamiya O et al. 2010 Int. J. Soc. Mater. Eng. Resour. 17182

[3] Saito A et al. 2014 Int. J. Soc. Mater. Eng. Resour. 20 181

[4] Suzuki T et al. 2017 Precis. Eng. 5032

[5] Sakamoto S et al. 2016 Key Eng. Mater. 70317

[6] Sakamoto S et al. 2017 Mater. Sci. Eng. 229012031

[7] Hayashi K et al. 1991 J. of the Soc. of Mater. Sci. 40 405

[8] Ueda K et al. 1982 Bull. of the Jpn. Inst. of Met. 21 225. 